\title{
Interference Mitigation in Wideband Radios using Spectrum Correlation and Neural Network
}

\author{
Andrea Toma*1,2, Tassadaq Nawaz ${ }^{1}$, Yue Gao ${ }^{2}$, Lucio Marcenaro ${ }^{1}$, Carlo S. Regazzoni ${ }^{1}$ \\ ${ }^{1}$ Dept. of Electrical, Electronic, Telecommunications Engineering and Naval Architecture - DITEN, University of Genoa, Italy \\ ${ }^{2}$ School of Electronic Engineering and Computer Science - EECS, Queen Mary University of London, UK \\ ${ }^{*}$ E-mail: andrea.toma@ginevra.dibe.unige.it
}

\section{A. Toma and T. Nawaz partecipated to the paper with equal contribution}

\begin{abstract}
Technologies such as Cognitive Radio and Dynamic Spectrum Access rely on spectrum sensing which provides wireless devices with information about the radio spectrum in the surrounding environment. One of the main challenges in wireless communications is the interference caused by malicious users on the shared spectrum.

In this manuscript, an artificial intelligence enabled (Al-enabled) cognitive radio framework is proposed at system-level as part of a Cyclic Spectrum Intelligence (CSI) algorithm for interference mitigation in wideband radios. It exploits the cyclostationary feature of signals to differentiate users with different modulation schemes and an artificial neural network as classifier to detect potential malicious users.

A dataset consisting of experimental modulated and dynamic signals is recorded by spectrum measurements with an in-house software defined radio testbed and then processed. Cyclostationary features are extracted for each detected signal and fed to a neural network classifier as training and testing data in a complex and dynamic scenario. Results highlight a classification rate of approximately 1 in most of cases, even at low transmission power. A comparison with two previous works with hand-crafted features, which employ an energy detector-based classifier and a naive Bayes-based classifier, respectively, is discussed.
\end{abstract}

\section{1}

\section{Introduction}

Recent advances in Software Defined Radio (SDR) and Machine Learning (ML), in addition to results obtained in computational neuroscience [1], open new horizons towards innovative technologies in different fields including Cognitive Radio (CR) [2]. Basically, CR deals with the growing demand and shortage of the wireless spectrum. In order to utilize the spectrum efficiently, TV white spaces (TVWS) [3-7] allows secondary users to use licensed spectrum bands provided that they change their access strategies to avoid or reduce interference to the primary users. Dynamic Spectrum Access (DSA), or dynamic channel access, assumes an important role in both TVWS cognitive radio networks [8] and the recent 5G technology [9].

On the other hand, though, signals can be transmitted in the shared spectrum with different objectives which means that legitimate signals use the spectrum in a legitimate way complying with the security requirements and avoiding interference to other users, while malicious signals are transmitted with the objective of causing interference or disrupting legitimate communications of the targeted system. Indeed, radio communications in wireless environments introduce security issues due to external attacks from malicious devices owing to the broadcast nature of radio propagation. In particular, the physical-layer (or PHY-layer) is extremely vulnerable to jamming attacks.

Nowadays, the need for measurable security in the context of interoperating services, applications, systems, and devices in a Cyber-Physical-Systems (CPSs) and Internet-of-Things (IoT) [10] framework requires the development of an appropriate paradigm. A step forward in that direction is made by SHIELD which consists of methodologies for building secure embedded systems [11]. Recently, the problem of malicious user detection has also been addressed by TVWS research in [12].

Spectrum sensing information plays a key role in anti-jamming systems. This information may be used to detect potential jamming entities [13] and to take proactive measures, as the channel hopping strategy in [14], to ensure communication continuity and security [15]. Moreover, a history of observations can be maintained and used to devise more effective anti-jamming tactics.

In literature, various spectrum sensing techniques have been proposed for CRs [16], such as energy detector (ED) [17], cyclostationary feature detector (CFD) [18], matched filtering detector (MFD) [19], and wavelet transform (WT)-based detector [20]. Among these methods, the CFD is capable of detecting the primary signal from the interference and noise even in very low signal-to-noise ratio (SNR) regions. This detection performance is achieved at the cost of increased implementation complexity. The Federal Communications Commission (FCC) of the United States has suggested CFD as an alternative to improve the detection sensitivity in CR networks. Generally, energy detector fails at low SNRs while matched filtering detector requires a dedicated receiver structure which may not be possible in a practical cognitive radio terminal. CFD exploits the cyclostationarity of modulated signals by detecting spectral peaks in spectral correlation function (SCF) or spectral coherence function (SOF) [21-23], which are sparse in both angular $(f)$ and cyclic $(\alpha)$ frequency domain. Major advantage of CFD based detector lies on its abilities to perform better than energy detector at low SNR values and to distinguish different modulated signals. Furthermore, the cyclic spectral analysis has been used as a robust tool for signal classification when the carrier frequency and bandwidth information is unavailable $[24,25]$. Compressed sensing (CS), investigated in wideband (WB) cognitive networks [26], has also been applied to a cyclostationary based detector in [27]. A comparison among the most common sensing methods (energy detection, cyclostationary, radio identification, match filtering, and waveform-based sensing) in terms of complexity and accuracy is made in [28]. Features-based algorithms require classification methods to evaluate the most probable class where the observed features belong to. Choosing the most suitable classification algorithm is not the only challenge to obtain satisfying performance and classification accuracy. Indeed, selection 


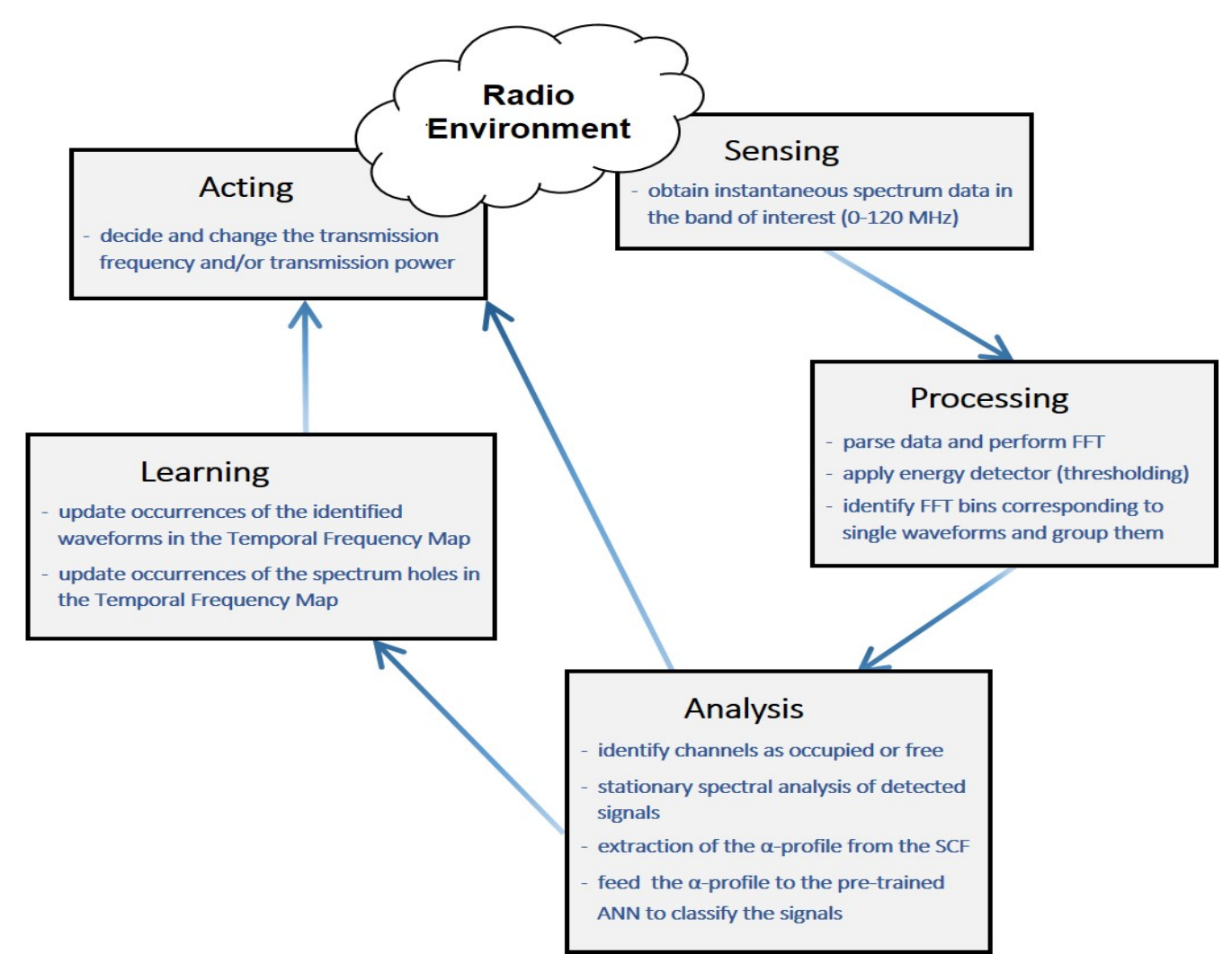

Fig. 1: Proposed artificial intelligence enabled (AI-enabled) cognitive radio framework with CSI algorithm.

of features and the feature extraction algorithm play a fundamental role as they strongly influence the accuracy on the classified signals. Spectrum intelligence algorithms in [29] and [30] utilize hand-crafted features such as bandwidth, magnitude, and variance for each of the signals. The former employs an ED-based classifier with a hypothesis test, while the latter proposes a Bayes-based classifier. The main drawback related to these two approaches is that both the variability of the extracted features and noise prevent to obtain accurate classification especially at low SNR level.

A Cyclic Spectrum Intelligence algorithm, Fig. 1, is proposed in this manuscript in an AI-enabled cognitive radio framework as an alternative to the existing solutions in $[29,30]$ and based on a cognitive cycle to acquire self-awareness from spectrum measurements. Firstly, instantaneous spectrum data is collected at sensing stage through a SDR testbed and, then, the wideband spectrum is processed to identify fast Fourier transform (FFT) bins corresponding to single waveforms and group them. Afterwards, analysis is applied. Unlike the spectrum intelligence in [29, 30], CSI employs a CFD to extract more advanced features and an Artificial Neural Network (ANN) [31] to classify the observed signals and perform interference mitigation in a WB spectrum. Specifically, the cyclostationary feature, called $\alpha$-profile, of each detected signal in the band-of-interest is classified through the ANN-based recognizer. Results are promising and show high classification rate even for low transmission power case. Moreover, CSI produces better or comparable classification rates with respect to the existing approaches. Cyclic spectral analysis and complexity are discussed throughout the manuscript. For the sake of completeness, learning and acting blocks are also introduced.

The main novelty is then the newly proposed analysis block as part of a new CSI algorithm for interference mitigation in wideband radios at system level. This improves the resulting performance of two major previous works in the literature for spectrum intelligence algorithms. Moreover, unlike previous work, our classification scheme is tested in a complex dynamic environment which is the most probable scenario in CR applications.
The remainder of the paper is organised as follows: the proposed CSI algorithm and the experimental set-up for the sensing block are described in Sec. 2. Data acquisition and the processing block are introduced in Sec. 3; while cyclostationary feature analysis and the neural network classifier in the analysis block are described in Sec. 4 which also includes a discussion on cyclic spectral analysis and complexity. In Sec. 5, Learning and Acting blocks conclude the cognitive cycle. Validation of the proposed approach with experimental data is analysed in Sec. 6 along with a comparison with two previous works. Some conclusive considerations and future work can be found in Sec. 7.

\section{Cyclic Spectrum Intelligence (CSI) algorithm and experimental set-up}

The principal idea behind the CSI algorithm is to continuously monitor relevant radio-frequency (RF) spectrum activities, identify potential threats to the communication, and take proactive measures to ensure communication robustness and secrecy. For doing so, the algorithm relies on the reliable spectrum sensing mechanism, correct identification and extraction of the relevant parameters, and secure software unsubjected to tampering. In comparison with the spectrum intelligence algorithm in [29], the proposed CSI algorithm employs a cyclostationary feature algorithm to extract the $\alpha$-profile feature from the detected signals which is then fed to a neural network to classify the waveforms present into the observed spectrum.

The functional process of the CSI algorithm can be represented in the form of the Cognitive Cycle, as shown in Fig. 1, consisting of 5 blocks: Sensing, Processing, Analysis, Learning, and Acting. Sensing and Acting blocks represent the interface with the external radio environment. The Cognitive Cycle is described throughout the manuscript.

At the sensing phase, data acquisition systems consisting of physical devices such as antennas, sensors and processing units are used as interface. Hardware architectures have been used in the literature to implement spectrum sensing in cognitive radio systems, such as the 


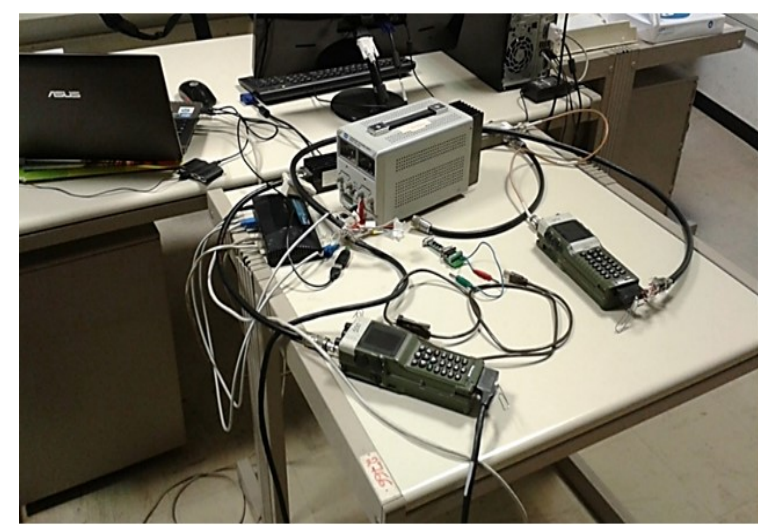

Fig. 2: SDR testbed utilised to generate the dataset with wideband spectrum measurements: hardware platform.

universal software radio peripheral (USRP) in a GNU-Radio framework in $[32,33]$. While, to validate the proposed CSI algorithm, a SDR hardware platform has been employed to generate modulated signals in a specified band, namely $0-120 \mathrm{MHz}$ which includes the Very High Frequency (VHF) band at 30-88 MHz. Alternatively, the Ultra High Frequency band (UHF) band can be selected. The testbed is remotely controlled and can be employed in on-line applications. Although previous work based on the aforementioned testbed was carried out in anti-jamming scenarios such in [30], it has never been collected a large amount of experimental data in dynamic environments in which signals change either their carrier frequency or their transmission power (or both of them), stored in a complex dataset with a wide range of signal characteristics and shapes, and organized for off-line applications.

The testbed employed to generate real-data is a SDR platform which consists of two Secure Wideband Multi-role - Single-Channel Handheld Radios (SWAVE HHs) shown in Fig. 2, the first one is the transmitter while the second one receives the wideband signal, connected through a dual directional coupler.

The fully operational SDR radio terminal SelexES (2013), SWAVE HH (from now on referred to as $\mathrm{HH}$ ), is capable of generating a multitude of wideband and narrowband waveforms. Currently, two functional waveforms are installed on the radio: SelfNET Soldier Broadband Waveform (SBW), whose channel bandwidth is adjustable in the range $1.25 \mathrm{MHz}$ to $5 \mathrm{MHz}$ with channel spacing of $2 \mathrm{MHz}$ and data is modulated using a fixed digital modulation technique, and VHF/UHF Line Of Sight (VULOS), which supports two analog modulation techniques, Amplitude Modulation (AM) and Frequency Modulation (FM), while both channel bandwidth and channel spacing are adjustable up to $25 \mathrm{kHz}$ [34].

The radio provides operability in both VHF (30 - 88 MHz) and UHF (225 - $512 \mathrm{MHz}$ ) bands. When VHF is selected, analog to digital conversion is performed directly at RF and the frequency band scanned is always $0-120 \mathrm{MHz}$; while in the case of UHF, the conversion is performed at intermediate frequency (IF) and the frequency band scanned depends on the center carrier frequency $f_{c}$ of the radio $\left(\left[f_{c}-35 ; f_{c}+85\right] \mathrm{MHz}\right)$. No selective filtering is applied before analog-to-digital conversion. Broadband digitized signal is then issued to the field-programmable gate array (FPGA), where it undergoes digital down conversion, matched filtering and demodulation.

Several interfaces are provided by the hypertach expansion placed at the bottom of each $\mathrm{HH}$, specifically, 10/100 Ethernet, USB 2.0, RS-485 serial, DC power interface $(\max 12.7 \mathrm{~V})$, and PTT. The software architecture of the radio is compliant with the Software Communications Architecture (SCA) 2.2.2 standard.

Maximum transmission power of the $\mathrm{HH}$ is $5 \mathrm{~W}$, with the harmonics suppression at the transmitter side over $-50 \mathrm{dBc}$. Superheterodyne receiver has specified image rejection better than $-58 \mathrm{dBc}$. Because of the high output power of the radios, one programmable attenuator is included in the communication path and programmed to their maximum attenuation value $-30 \mathrm{~dB}$.

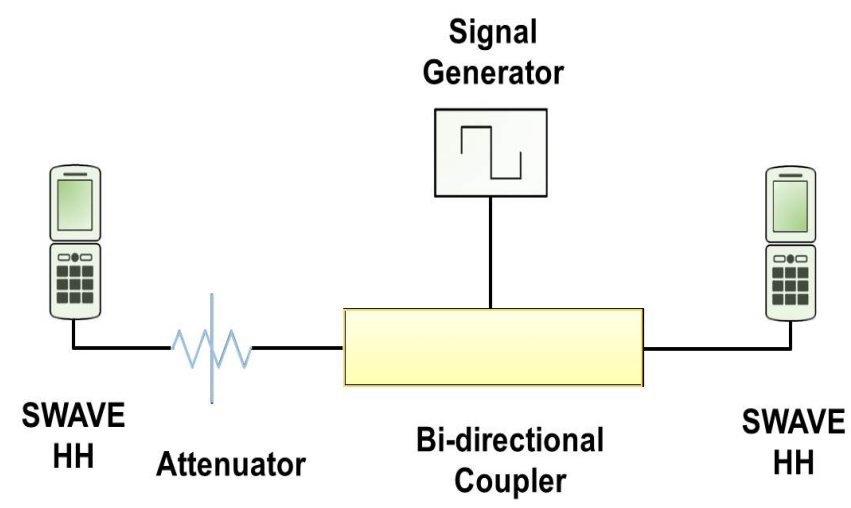

Fig. 3: Diagram of the main components of the testbed and their connections.

Agilent 778D 100MHz-2GHz dual directional coupler with 20dB nominal coupling is used as communication medium between the two HHs.

Guided propagation exhibits several important advantages with respect to the over-the-air implementation: accurate and stable RF levels, repeatability of the experiments without the uncertainties characteristic to wireless transmission, possibility to connect test instruments and generators, avoiding regulatory issues related to transmitting outside of the allowed frequency bands.

The testbed provides support for remote control of HH's transmission and reception parameters through Ethernet and the Simple Network Management Protocol (SNMP v3). The general diagram of the testbed is shown in Fig. 3. Full details on the testbed architecture may be found in [34].

Concerning the spectrum sensing process, the HH's 14-bit Analog-to-Digital-Converter (ADC) performs sampling at 250 Msamples/s. Every 3 seconds, a burst of 8192 consecutive samples is buffered, and then output over the serial port at 115200 bauds. The samples are then parsed and transformed into the frequency domain using the FFT. The bandwidth of the corresponding spectrum is $120 \mathrm{MHz}$ wide around the center carrier frequency of the radio. Consequently, the effective resolution is $29.3 \mathrm{kHz} / \mathrm{sample}$. In order to obtain higher frequency resolutions, two possible changes to the testbed are increasing the buffer size on the HHs, and finding ways to transfer spectrum data at higher baud rate. Further details can be found in [34].

\section{Data acquisition and processing}

Acquisition of the wideband RF spectrum, is performed periodically for the frequency band of interest. This may be done by taking either a quiet or an active approach, depending on the implementation of the architecture. Quiet approach implies that the radio is able to performing sensing simultaneously with transmitting/receiving, whereas in active sensing, the radio needs to stop transmitting/receiving while sensing takes place.

\subsection{Data Acquisition}

By means of the testbed described in the previous section, real-data is collected and stored in a dataset. To this end, the VHF transmission band where the radios are operable has been utilized, meaning that the spectrum sensing is performed for the frequency band of $0-120$ MHz.

The data consists of a large amount of spectrum observations containing a number of narrowband signals. More specifically, as shown in Fig. 4 (thin solid blue line), each spectrum consists of a SBW signal (digitally modulated signal) transmitted by the transmitting $\mathrm{HH}$ device and a number of signals (from the environment) such as the FM signal (in the $88-108 \mathrm{MHz}$ band) and an unknown signal at 


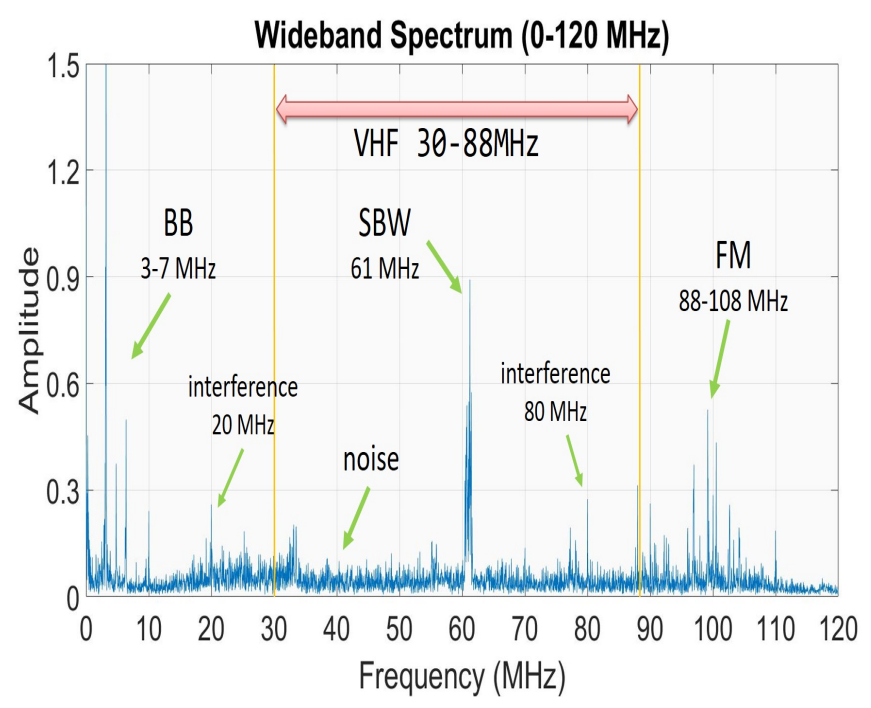

Fig. 4: Spectrum observation in the $0-120 \mathrm{MHz}$ band ( 1 burst) which includes the SBW signal at $61 \mathrm{MHz}$ and with transmission power equal to $-3 \mathrm{dBm}$ as well as other signals from external environment.

0-7 MHz. Two interference signals at $20 \mathrm{MHz}$ and $80 \mathrm{MHz}$, respectively, are also observed. Further details are given in Sec. 4. The parameters of the SBW signal can be set remotely and, in particular, the transmission power and the carrier frequency of the transmitted SBW signal have been considered which are given 3 different values (7dBm-full, $4 \mathrm{dBm}$-half, and $-3 \mathrm{dBm}$-one-tenth for the transmission power; $51 \mathrm{MHz}-61 \mathrm{MHz}-71 \mathrm{MHz}$ for the carrier frequency). Consequently, the dataset consists of spectrum measurements grouped in 9 different configurations, as shown in Table 1. Each configuration consists of more than 2500 bursts. The corresponding time-domain samples have also been stored in the dataset. The experimental step is discussed in Sec. 6.

\subsection{Processing}

This section describes the pre-processing block of real-data to detect frequency bins belonging to the different waveforms inside the spectrum. The main parameters for the pre-processing are also described.

First of all, the received spectrum observations are smoothed in the frequency domain through a simple moving average applied to the samples in order to reduce the sharp fluctuations due to noise which can be seen in each received spectrum.

Then, based on a sensible choice for a specific threshold, the background noise is eliminated, keeping only the FFT bins corresponding to actual signals. Basically, this process can be thought as an energy detection and formally corresponds to solving the decision problem between the following two hypotheses:

$$
Z(n)=\left\{\begin{array}{ll}
\eta(n) & H_{0} \\
S(n)+\eta(n) & H_{1}
\end{array} ; n=1, \ldots, N_{S}\right.
$$

where $Z(n), S(n)$ and $\eta(n)$ are the received signal, the transmitted signal and the noise samples, respectively. $H_{0}$ is the null hypothesis corresponding to the absence of the signal (in this case, received signal consists only of noise), and $H_{1}$ is the alternative hypothesis corresponding to the presence of the signal, while $N_{S}$ is the number of samples acquired during the sampling process.

Based on the Neyman-Pearson lemma, the most common approaches to finding the appropriate threshold are the Constant Detection Rate (CDR) and Constant False Alarm Rate (CFAR) detectors, where threshold is set adaptively depending on the SNR regime and the characteristics of the sensed wideband signal. However, even in adaptive thresholding, presence of interference may make the energy detector come to incorrect decisions.
Table 1 Configurations for the values of the transmission parameters (carrier frequency and transmission power) of the SBW signal in the collected dataset.

7dBm - Full Power

\begin{tabular}{|c|c|c|}
\hline $\boldsymbol{f}_{\boldsymbol{c}}=51 \mathrm{MHz}$ & $\boldsymbol{f}_{\boldsymbol{c}}=61 \mathrm{MHz}$ & $\boldsymbol{f}_{\boldsymbol{c}}=71 \mathrm{MHz}$ \\
\hline
\end{tabular}

4dBm - Half Power

\begin{tabular}{|c|c|c|}
\hline IV & $V$ & $V I$ \\
$\boldsymbol{f}_{c}=51 \mathrm{MHz}$ & $\boldsymbol{f}_{c}=61 \mathrm{MHz}$ & $\boldsymbol{f}_{c}=71 \mathrm{MHz}$ \\
\hline
\end{tabular}

-3dBm - Onetenth Power

\begin{tabular}{|c|c|c|}
\hline VII & VIII & $I X$ \\
$\boldsymbol{f}_{c}=51 \mathrm{MHz}$ & $\boldsymbol{f}_{c}=61 \mathrm{MHz}$ & $\boldsymbol{f}_{\boldsymbol{c}}=71 \mathrm{MHz}$ \\
\hline
\end{tabular}

In most applications, the analysed spectrum is underutilised (usage of licensed bands is an example $[35,36]$ ) which means that there is only a limited number of actual narrowband signals in the scanned wideband signal at any instant of time. In this scenario, suboptimal thresholding algorithms with low computational complexity, where CFAR or CDR performance is not necessarily achieved, can be considered. Indeed, it has been shown that the threshold $\hat{\delta}$ may be adaptively set based only on the mean value of the magnitudes of the scanned wideband signal [29], and given by:

$$
\hat{\delta}=2 \cdot \frac{1}{n} \sum_{n=1}^{N_{S}}|Z(n)|
$$

$K$ frequency bins are identified as a result of the thresholding process.

In a wideband and sparse spectrum observation there are $L$ actual signals $(K>L)$ each of them consists of a number of bins. For this reason, frequency bins corresponding to the same signal need to be grouped together. In high-SNR environments, consecutive samples can be grouped together and classified as single waveforms. However, in practical situations, some frequency bins may have erroneous magnitude values as a result of imperfect sampling and would thus be discarded during the thresholding phase. In this case, the bin grouping process considers two (o more) groups of consecutive bins as a single signal if they are close enough each other. More formally, the maximum acceptable distance (in $\mathrm{Hz}$ ) between the two samples belonging to the same waveform is defined as:

$$
d_{M A X}=M \cdot d_{f}
$$

$M$ is the maximum number of consecutive samples that could be erroneously disregarded, and $d_{f}$ is the frequency resolution of the FFT, given by:

$$
d_{f}=\frac{2 \cdot f_{\max }}{N_{S}}
$$

where $f_{\max }$ is the maximum resolvable frequency (which in case of Nyquist sampling equals to half of the sampling frequency). After the thresholding step, grouped waveforms undergo smoothing, in order to reduce impacts of the imperfect and erroneous sampling. For achieving this, a second stage moving average filter has been implemented. For a waveform that consists of $n G$ grouped bins with magnitudes $S_{1}, \ldots, S_{n G}$, filtering with the window length $W$ results in the filtered bins given by [30]:

$$
S_{f}\left(n_{i}\right)=\frac{1}{P} \sum_{j=i-P+1}^{i} S_{j} ; \quad i=P, \ldots, n G
$$

Consequently, each element in Eq. (5) is an average of the preceding $P$ points. 


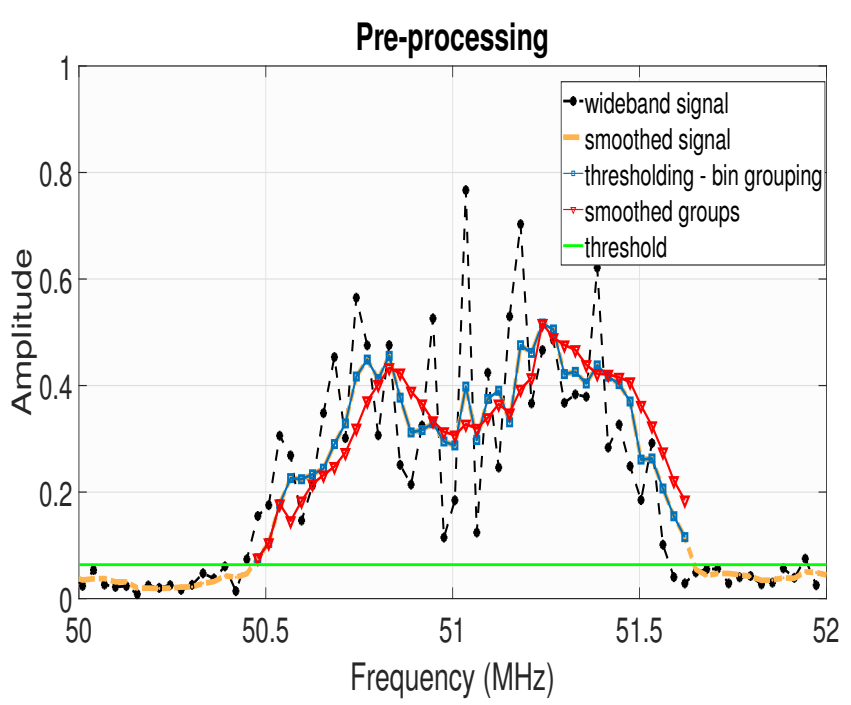

Fig. 5: Pre-processing applied to the WB signal (in both the pictures, only the SBW signal is shown): (a) WB signal, smoothed signal, thresholding and bin grouping, and threshold; (b) also includes the waveform after group smoothing.

Fig. 5 illustrates the difference between the original transmitted SBW signal, the corresponding smoothed signal, the sensed FFT bins, and the estimated signal after performing thresholding/bin grouping. The smoothed group corresponding to the SBW signal is also shown.

A comparison between the original wideband signal (thin dotted blue line) and the corresponding signal after having undergone the pre-processing (thick dash-dotted red line) is made in Fig. 6.

This concludes the frequency domain pre-processing phase which is applied to the collected wideband signals.

After this phase, the inverse Fourier transform is applied to produce the corresponding time domain signals from the pre-processed wideband spectrum measurements and, then, the CFD algorithm produces the $\alpha$-profile from the time-domain sub-signals as described in the following section.

\section{Cyclostationary feature analysis and neural network}

In this section, the classification process is described in order to present an application of the dataset to theoretical framework. After the pre-processing phase in Sec. 3.2, the waveforms are classified as either "potentially malicious" (PM) or "friendly" (FR). The former class refers to signals which aim to disrupt or degrade communications among legitimate users (belonging to the latter class). In the $0-120 \mathrm{MHz}$ wideband spectrum, the jammer is supposed to be the SBW signal (which is capable of changing its transmission parameters), while legitimate waveforms as in Fig. 6 are the base-band (BB) signal, interference at $20 \mathrm{MHz}$ (we call it medium-band, MB, interference) and $80 \mathrm{MHz}$ (we call it high-band, $\mathrm{HB}$, interference), and the FM signal. In order to differentiate them, a cyclostationary feature based algorithm with an ANN as classifier is applied to the dataset.

\subsection{Cyclostationary feature analysis}

When signal processing techniques assume communication signals as being stationary, their statistical parameters such as mean and variance do not vary with time. In this case, signals are modelled as one-dimensional (1D) autocorrelation function and the corresponding power spectrum density (PSD) can be obtained through Fourier transform of the autocorrelation function [37]. On the other hand, most of the signals in communication systems are in fact

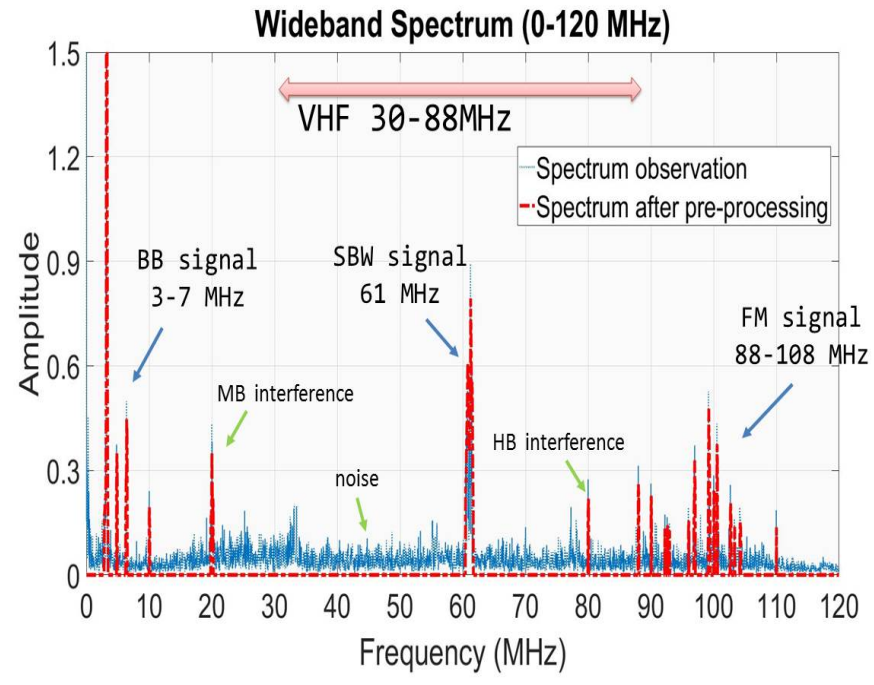

Fig. 6: Wideband spectrum measurement before pre-processing (thin dotted blue line) and after pre-processing (thick dash-dotted red line).

cyclostationary [38]. This means that their statistical parameters are periodically or cyclically stationary. A two-dimensional (2D) autocorrelation function is used to model cyclostationary signals where the additional dimension is the cycle frequency, denoted as $\alpha$, at which the 1D autocorrelation function is computed. For each $\alpha$, a cyclic-spectrum-cut is produced which is a function of the parameter $\alpha$ and denoted as $S_{x}^{\alpha=f_{0}}(f)$, namely a cyclic-spectrum-cut at $\alpha=f_{0}$. Considering the whole set of values for $\alpha$, a threedimensional (3D) cyclic spectrum function is obtained where the three dimensions are: $f, \alpha$, and magnitude of the SCF. When $\alpha=0$, the cyclic-spectrum-cut corresponds to the conventional PSD since the spectrum completely correlates with itself.

Specifically, a process $x(t)$ is said to be wide-sense cyclostationary with period $T_{0}$ if its mean $E[x(t)]=\mu_{x}(t)$ and autocorrelation $E\left[x(t) x^{*}(t+\tau)\right]=R_{x}(t, \tau)$ are both periodic with period $T_{0}$, in such case, they can be defined respectively as:

$$
\mu_{x}\left(t+T_{0}\right)=\mu_{x}(t) ; \quad R_{x}\left(t+T_{0}, \tau\right)=R_{x}(t, \tau) .
$$

The autocorrelation function of a wide-sense cyclostationary process can be expressed in terms of its Fourier series components:

$$
R_{x}(t, \tau)=\sum_{\alpha} R_{x}^{\alpha}(\tau) e^{j 2 \pi \alpha t}
$$

where $\alpha=\frac{a}{T_{0}}$ with $a$ integer, $E[\cdot]$ is the expectation operator, $\alpha$ is the set of Fourier components, and $R_{x}^{\alpha}(\tau)$ represents the cyclic autocorrelation function (CAF) and gives Fourier components. CAF is given by:

$$
R_{x}(\tau)=\lim _{T \rightarrow \infty} \frac{1}{T} \int_{\frac{-T}{2}}^{\frac{T}{2}} R_{x}(t, \tau) e^{-j 2 \pi \alpha t} d t .
$$

When $R_{x}(t, \tau)$ is periodic in $t$ with period $T_{0}$, (7) can be expressed as:

$$
R_{x}^{\alpha}(\tau)=\frac{1}{T_{0}} \int_{\frac{-T_{0}}{2}}^{\frac{T_{0}}{2}} R_{x}(t, \tau) e^{-j 2 \pi \alpha t} d t .
$$

The Fourier Transform of the CAF is known as SCF and is given by:

$$
S_{x}^{\alpha}(f)=\int_{-\infty}^{\infty} R_{x}^{\alpha}(\tau) e^{-j 2 \pi f \tau} d \tau
$$

where $\alpha$ is the cyclic frequency and $f$ the angular frequency. The major benefit of spectral correlation is its insensitivity to background 
Interference Mitigation

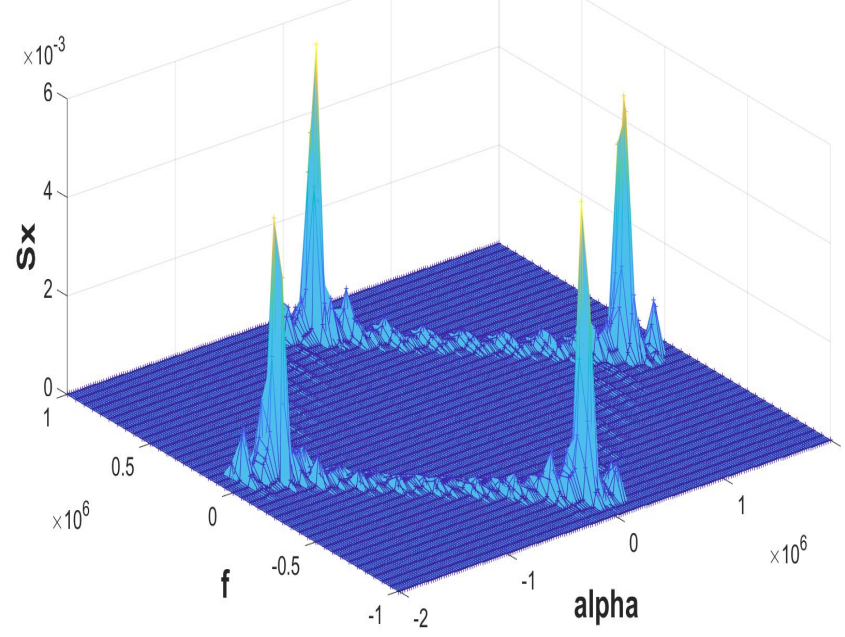

(a) $\mathrm{SCF}$ of the 3-7 MHz BB signal
Interference Mitigation

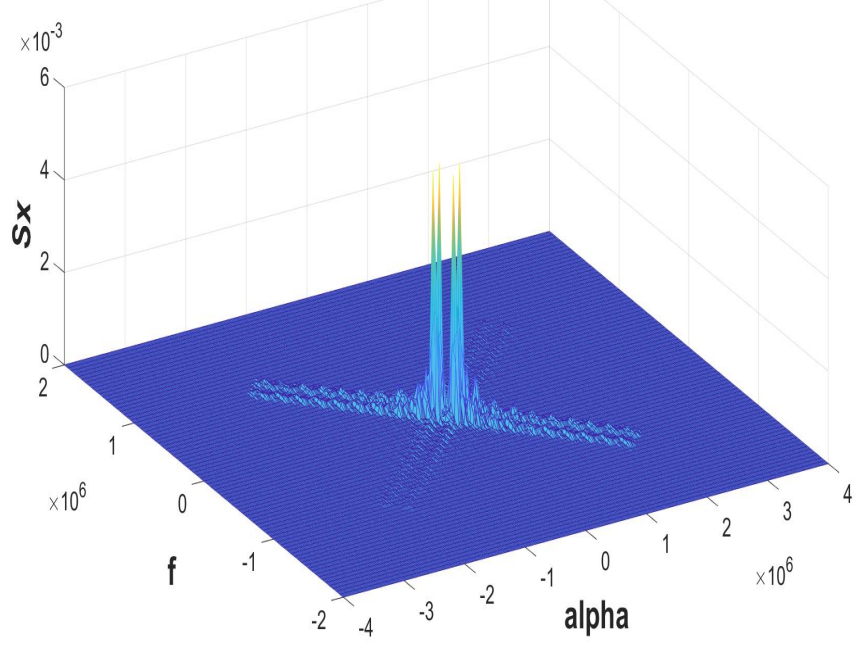

(b) SCF of the SBW signal

Fig. 7: SCF of two of the detected signals in the WB spectrum: (a) BB signal and (b) SBW signal.

noises. Since the temporal correlation of different spectral components are measured, and the spectral components of noise are completely uncorrelated in time due to the fact that noise is widesense stationary process, such noise does not play significant factor in the SCF. In other words, the existence of correlation between separated spectral components, with separation equal to $\alpha$, is called spectral redundancy [37] which is found in cyclostationary signals, while it is not usually observed in noise. This fact allows the spectral correlation of a signal to be accurately calculated even at low SNRs. Furthermore, different types of modulated signals - AM, FM, phase shift keying (PSK), frequency shift keying (FSK), minimum-shift keying (MSK), quadrature amplitude modulation (QAM), pulseamplitude modulation (PAM), and so forth - with overlapping power spectral densities have highly distinct SCFs.

The SCF of the SBW signal and the 3-7 MHz BB signal in the 0-120 MHz spectrum are shown in Figs. 7(a) and 7(b), respectively.

Since SCF computation requires large amount of data, which makes it unreasonable for a classifier to operate on it in real time, the cycle frequency profile ( $\alpha$-profile) has been employed in this paper as feature for classification. Specifically, the $\alpha$-profile of SCF for a signal $x$ is given by:

$$
I(\alpha)=\max _{f}\left[S_{x}^{\alpha}\right]
$$

The $\alpha$-profile of signals in the wideband spectrum of interest is shown in Sec. 6, while a more detailed description about SCF of some modulated signals can be find in Sec. 4.3 addressing the theoretical framework.

\subsection{Neural network classifier}

Our proposed system uses an ANN as classifier due to its ease of implementation and potential to generalize any carrier frequency, symbol rate and phase offset. The system was designed to classify PM and FR signals. We trained the ANN to identify the two classes of signals defined at the beginning of this section. The SCF of the detected signals produces a large amount of data, which makes impossible for a classifier to work on it in real time. In order to reduce the amount of data for a classification stage, we used the $\alpha$-profile as input feature for an ANN. Accordingly, the proposed ANN in Fig. 8 is composed of $I$ inputs related to the dimensionality of $\alpha$-profile, a single hidden layer whose $N$ neurons use the hyperbolic tangent sigmoid as neural transfer function, and an output layer of two neurons related to each class of signal considered

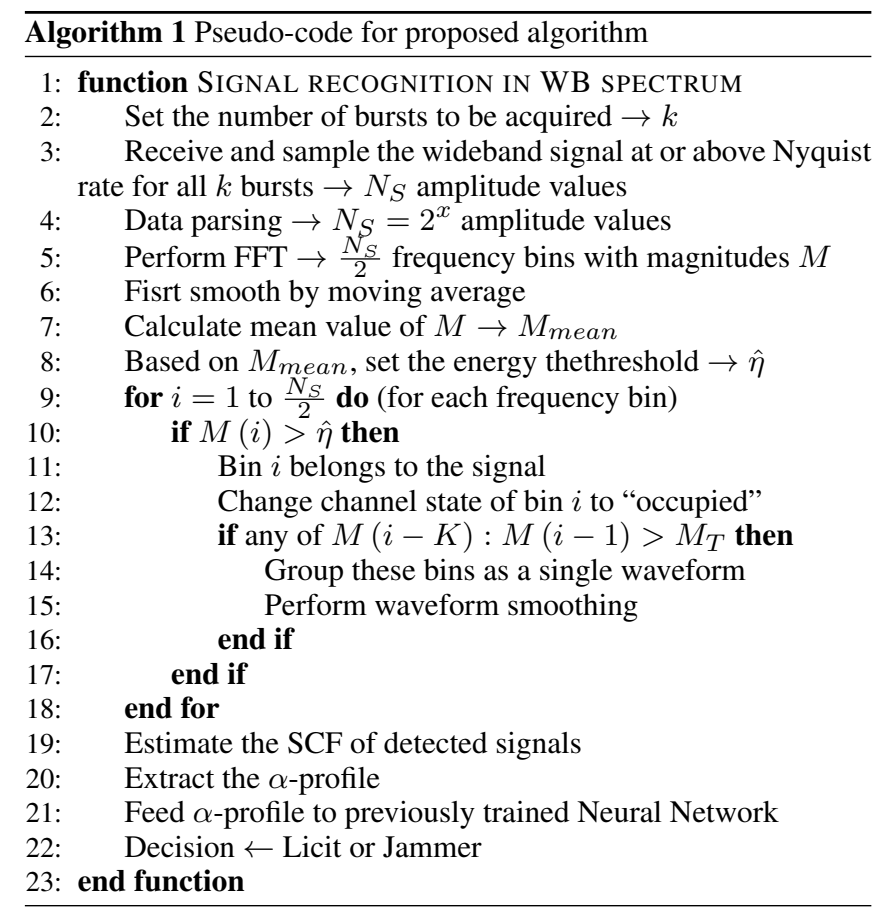

in this work. Each output value is in the range $(0,1)$. Accordingly, the output class with the highest value between $(0,1)$ is considered as the signal class. An ANN training based on the scaled conjugate gradient (SCG) back-propagation [39] is adopted.

The selection of a single hidden layer is proposed due to the classification process simplicity of this particular problem, it was found that with a single layer results over the $99 \%$ of true positive classification were obtained for the 2 types of signal classes considered in this work. Employing more hidden layers would increment the training time and overall results would not be significantly improved.

The corresponding pseudo-code of the proposed algorithm is outlined in Algorithm 1.

It can be summarized as follows: the receiver observes a WB signal and then energy detection and pre-processing are performed. The $\alpha$-profile of SCF for each detected sub-signal is subsequently extracted. After that, detected signals go through the classification 


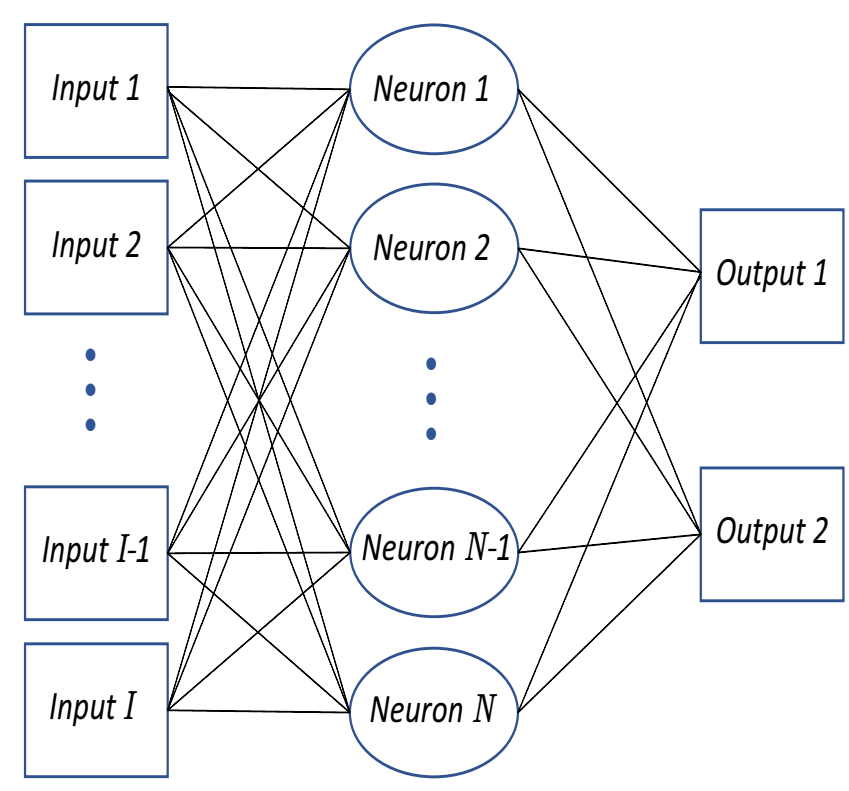

Fig. 8: Proposed Artificial Neural Network used as classifier in the CSI algorithm with $I$ inputs, one hidden layer with $N$ neurons, and 2 outputs.

process. The $\alpha$-profile of detected signals are fed to a previously trained ANN. The ANN classifies the received narrowband signal, in the WB spectrum, as either a licit or a potential malicious user.

\subsection{Cyclic spectral analysis}

Theoretical analysis of cyclic spectrum for a number of different modulation types (both analog and digital) can be found in the literature [40-42]. From the spectrum representation in Fig. 6, signals with different modulation schemes can be observed. For example, the BB signal found at low-frequency band 3-7 MHz is an AM signal, while the SBW is a digitally modulated signal with quaternary-PSK (QPSK) scheme. The two interferences are also digital modulated signals. Finally, the FM signal is observed on the right side of the spectrum. In the following, SCF of QPSK, AM, and FM signals is detailed as an example.

QPSK: a PSK signal can be thought either as a binary amplitudeshift keying (ASK) for $M=2$ or as a QAM when $M>2(M$ is the number of points in the signal constellation), namely

$$
m(t)=u_{I}(t) \cos \left(2 \pi f_{0} t+p_{0}\right)-u_{Q}(t) \sin \left(2 \pi f_{0} t+p_{0}\right)
$$

with in-phase and quadrature components $u_{I}(t)$ and $u_{Q}(t) . f_{0}$ is the carrier frequency and $p_{0}$ is a phase factor. In the specific case of QPSK signal, phases are separated by $\pi / 2 \mathrm{rad}$ and the in-phase and quadrature components are time-aligned as follows

and

$$
u_{I}(t)=\sum_{n=-\infty}^{\infty} u_{n}^{I} q\left(t-n T_{0}-t_{0}\right)
$$

$$
u_{Q}(t)=\sum_{n=-\infty}^{\infty} u_{n}^{Q} q\left(t-n T_{0}-t_{0}\right)
$$

respectively; the keying rate is defined by $1 / T_{0}$, while $t_{0}$ is a fixed pulse-timing phase parameter. The carrier function $q(t)$ is a deterministic finite-energy pulse and modulated by $u_{n}^{I}$ and $u_{n}^{Q}$ which are random sequences assumed to be purely stationary. Consequently, the SCF can be written as [41]:

$$
S_{m}^{\alpha}(f)=\frac{1}{2 T_{0}}\left[Q\left(f+\alpha / 2+f_{0}\right) Q\left(f-\alpha / 2+f_{0}\right) S_{u_{I}}^{\alpha}\left(f+f_{0}\right)\right.
$$

$$
\left.+Q\left(f+\alpha / 2-f_{0}\right) Q\left(f-\alpha / 2-f_{0}\right) S_{u_{Q}}^{\alpha}\left(f-f_{0}\right)\right] e^{-i 2 \pi \alpha t_{0}}
$$

where the in-phase and quadrature components are assumed to be balanced such that $S_{u_{I}}^{\alpha}(f)-S_{u_{Q}}^{\alpha}(f) \equiv 0$. In this way, $S_{u_{I}}^{\alpha}(f)=$ $S_{u_{Q}}^{\alpha}(f)=1$ if $\alpha=k / T_{0}$, and $S_{u_{I}}^{\alpha}(f)=S_{u_{Q}}^{\alpha}(f)=0$ if $\alpha \neq$ $k / T_{0}$. The function $Q(f)$ is defined in [40] for a linear periodically time-variant system.

$\boldsymbol{A M}$ : when a random lowpass signal $a(t)$ with PSD $S_{a}(f)$ is used to modulate the amplitude of a sine wave, the resulting amplitude modulated signal $m(t)$ is given by [43]

$$
m(t)=a(t) \cos \left(2 \pi f_{0} t+p_{0}\right)
$$

and the general formula of its SCF is given by

$$
S_{m}^{\alpha}(f)= \begin{cases}\frac{1}{4} e^{ \pm i 2 p_{0}} S_{a}(f), & \text { if } \alpha= \pm 2 f_{0} \\ \frac{1}{4} S_{a}\left(f+f_{0}\right)+\frac{1}{4} S_{a}\left(f-f_{0}\right), & \text { if } \alpha=0 \\ 0, & \text { otherwise }\end{cases}
$$

FM: in many cases, the transmitted signal is modulated as

$$
m(t)=a \cos \left(2 \pi f_{0} t+\varphi(t)\right)
$$

where $\varphi(t)$ is the phase of the FM signal, $a$ is the carrier amplitude, and $f_{0}$ is the carrier frequency. The corresponding SCF is given by [44]

$$
S_{m}^{\alpha}(f)= \begin{cases}\frac{1}{4}\left[\Psi_{r}(f)\right] e^{ \pm i 2 \varphi_{0}}, & \text { for } \alpha= \pm 2 f_{0} \\ \frac{1}{4}\left[\Psi_{r}\left(f+f_{0}\right)+\Psi_{r}\left(f-f_{0}\right)\right], & \text { if } \alpha=0 \\ 0, & \text { otherwise }\end{cases}
$$

where $\Psi_{r}(f)$ is the Fourier transform of the joint characteristic function for $\varphi(t+\tau / 2)$ and $\varphi(t-\tau / 2)$ given in [37].

Further details and SCF of other modulation schemes (both analog and digital) can be found in [37, 40, 41, 43, 44].

\subsection{Complexity analysis}

SCF computation complexity: efficient algorithms to compute SCF are defined as FFT time smoothing algorithms, In this work, the strip spectral correlation algorithm (SSCA) has been considered. Basically, SSCA is computed by multiplying the complex envelope with the conjugate of the received signal. The corresponding block diagram is discussed in [45] where 2 FFT blocks are employed. The complex envelope is a function of the frequency $f$, thus, the number of the first FFT points $N^{\prime}$ is inversely related to the frequency resolution $\Delta f$. While, the SCF formula is a $2 \mathrm{D}$ function in terms of $f$ and $\alpha$ and the number of the second FFT points $N$ is inversely proportional to the cyclic frequency resolution $\Delta \alpha$. This mean that an increase in the values of $\Delta f$ and $\Delta \alpha$ will result in larger computational complexity although random effects are reduced and, consequently, the SCF reliability increases. The complexity of the SSCA algorithm is summarised in Tab. 2 in terms of the number of complex multiplications required to estimate the cyclic cross spectrum of two complex signals [45].

The total complexity is then given by $N^{\prime} N\left(3+\frac{1}{2} \log _{2} N^{\prime} N\right)$. The SSCA is a highly parallel algorithm.

ANN computation complexity: a feedforward neural network is characterised by the total number of weights and biases, $N$, which form a weight vector $\mathbf{w}$. In the conventional back-propagation (BP) algorithm, a global error function $E(\mathbf{w})$ can be computed with one forward step, while its gradient $E^{\prime}(\mathbf{w})$ with one forward and one backward step. The complexity of calculating the error function and its gradient is $O\left(N^{2}\right)$ and $O\left(3 N^{2}\right)$, respectively. The BP algorithm often behaves very badly on large-scale problems and user dependent parameters, like learning rate, determine its performance. 


\begin{tabular}{c|lc}
\hline $\begin{array}{l}\text { Computational } \\
\text { section }\end{array}$ & $\begin{array}{l}\text { Data } \\
\text { tapering }\end{array}$ & $N^{\prime}$-FFT \\
- SSCA & $N^{\prime} N$ & $\frac{N^{\prime} N}{2} \log _{2} N$ \\
\hline
\end{tabular}

\begin{tabular}{ccc}
\hline $\begin{array}{l}\text { Down- } \\
\text { conversion }\end{array}$ & $\begin{array}{c}\text { Sequences } \\
\text { multiplication }\end{array}$ & $N$-FFT \\
$-N^{\prime} N$ & $N^{\prime} N$ & $\frac{N^{\prime} N}{2} \log _{2} N$ \\
\hline
\end{tabular}

Alternatively, conjugate direction methods, such as the conjugate gradients (CG) method, exploit second order approximation of the error function which, in addition to the gradient, utilizes the Hessian matrix denoted as $E^{\prime \prime}(\mathbf{w})$. There exists a unique global minimum only if the Hessian matrix is positive definite. But in many cases, it has shown to be indefinite in different areas of the weight space and the CG fails in the attempt to minimize the error function. Further details can be found in [39] which also proposes the scaled conjugate gradient back-propagation algorithm. As mentioned in Sec. 4.2, the SCG is adopted to train the ANN used as classifier. In this case, a calculation complexity per iteration of $O\left(7 N^{2}\right)$ has been determined. When the algorithm is implemented, this complexity can be reduced to $O\left(6 N^{2}\right)$.

\section{$5 \quad$ Learning and acting algorithms}

After the new Analysis block proposed in this manuscript and belonging to the cognitive cycle, Leaning and Acting blocks presented in [30] conclude the proposed CSI algorithm.

\subsection{Learning}

After having identified occupied channels and spectrum holes in the 0-120 MHz band, and classified the detected signals through the cyclostationary feature algorithm with an artificial neural network as classifier, the CSI algorithm is thought to include a learning process strategy based on a Temporal Frequency Map which summarises previous occurrences of waveforms inside the band-of-interest, Fig. 9. The $0-120 \mathrm{MHz}$ band is divided into $n$ channels. In each cycle of the cognitive strategy, the proposed algorithm accesses the Temporal Frequency Map which is a $n \times 3$ matrix where the number of occurrences of FR waveforms $\left(m_{F R}\right)$, PM waveforms $\left(m_{P M}\right)$, defined in Sec. 4 , and spectrum holes $\left(m_{S H}\right)$ for each of the $n$ channels are stored. Then previous values are updated with the newly acquired and processed information. The learning process can implement temporal forgiveness in order to include into the computation only the spectrum activities corresponding to the last $k$ spectrum observations which are used to predict future activities and make suitable decisions. This reduces the probability of data becoming obsolete, at the expense of the lower amount of accessible information.

\subsection{Acting}

Finally, based on the processed spectrum information, current transmission parameters (channel and transmission power) and the history obtained from the Temporal Frequency Map, the CR device may decide to act in order to improve its chances of reliable transmission. The actions consist of proactive changes of the transmission frequency (channel surfing), or the transmission power whenever a threat is detected. The system is considered "under threat" when a "potentially malicious" waveform is identified on the channel close to the channel currently used for transmission. The new channel for the transmission is then chosen according to:

$$
c_{t+1} \in\left(c_{t}=S H \mid X\left(c_{t}\right)=\min \right) .
$$

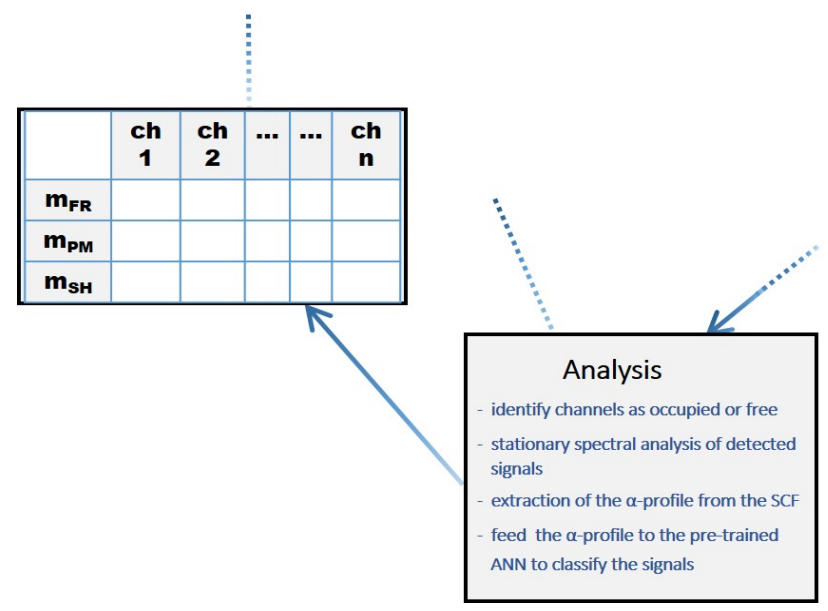

Fig. 9: Temporal Frequency Map in the Learning block which summarises previous occurrences of waveforms inside the band-ofinterest.

This means that the new channel $c_{t+1}$ is selected among all the channels $c_{t}$ that are currently spectrum holes, such that the $X\left(c_{t}\right)$ is minimum. $X\left(c_{t}\right)$ represents the expected channel occupancy, defined as:

$$
X\left(c_{t}\right)=l^{2} \cdot m_{P M / c_{t}}+(l+1) \cdot m_{F / c_{t}}-m_{S H / c_{t}},
$$

where $m_{P M / c_{t}}, m_{F R / c_{t}}$ and $m_{S H / c_{t}}$ represent the occurrences of the PM waveforms, FR waveforms, and spectrum holes on the channel $c_{t}$ over the last $l$ steps, respectively. The coefficients $l^{2}$ and $(l+1)$ are assigned in order to give highest priority to actions which avoid channels with history of occurrences of PM waveforms, followed by the channels with history of occurrences of FR waveforms. In this way, it is ensured that each possible channel occupancy corresponds to a unique combination of FR waveforms, PM waveforms, and spectrum holes. The new transmission power is chosen according to:

$$
P_{t+1} \in P \quad \text { s.t. } \quad P>10 \log _{10} \hat{\delta}+3 d B .
$$

where $\hat{\delta}$ is the threshold of the energy detector defined in Eq. (2).

Further applications include the capability of the cognitive system to learn from the actions of a human operator through a graphical user interface (GUI) allowing the human operator to overrule the decision of the cognitive algorithm and change transmission parameters such as the operating frequency and the transmission power. The role of the GUI is then to allow the human operator to take decisions irrespectively of the decisions of the CSI algorithm. However, it also presents an interesting motivation for considering principles of cognitive refinement, i.e., refining the reasoning behaviour of the algorithm, which is currently policy-based, by learning from the actions of the human operator.

\section{Validation of the proposed algorithm}

In order to evaluate the performance of the newly introduced analysis block of the cognitive cycle in the CSI algorithm (based on spectral correlation detector and neural network classifier for interference mitigation) a set of experiments is performed using the software defined radio testbed architecture described in Sec. 2. The sampling rate is set at Nyquist rate for each type of detected signals. In our experiments, the SBW signal represents the "potentially malicious" waveform and is transmitted by the transmitting HH. Its transmission parameters are given different values according to the Table 1 in Sec. 3.1, namely the carrier frequency and the transmission power of the SBW signal assume one among the nine configurations with full, half, and one-tenth transmission power. All other detected signals mentioned in Sec. 3.1 are considered as "friendly". The objective of this section is to show the performance 


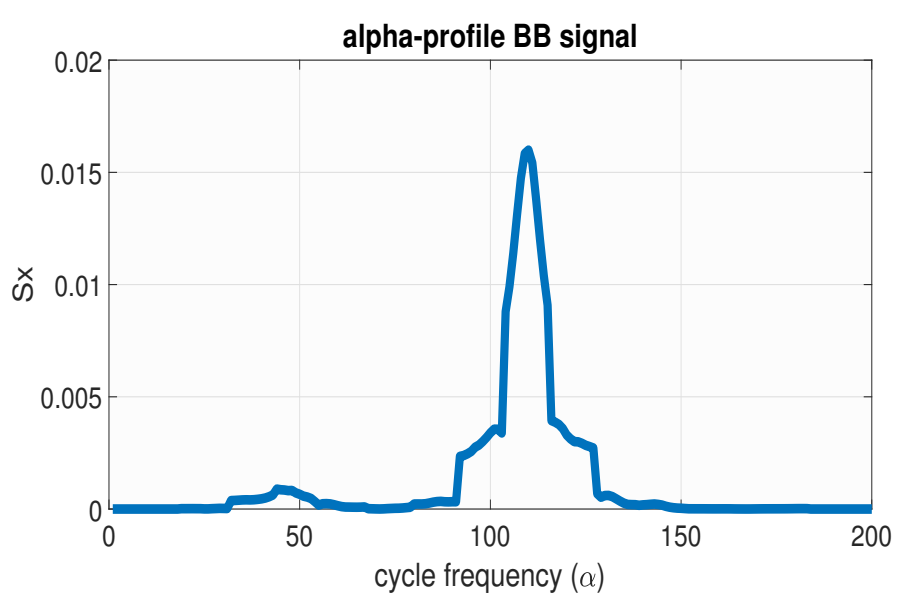

(a) 3-7 MHz signal - BB

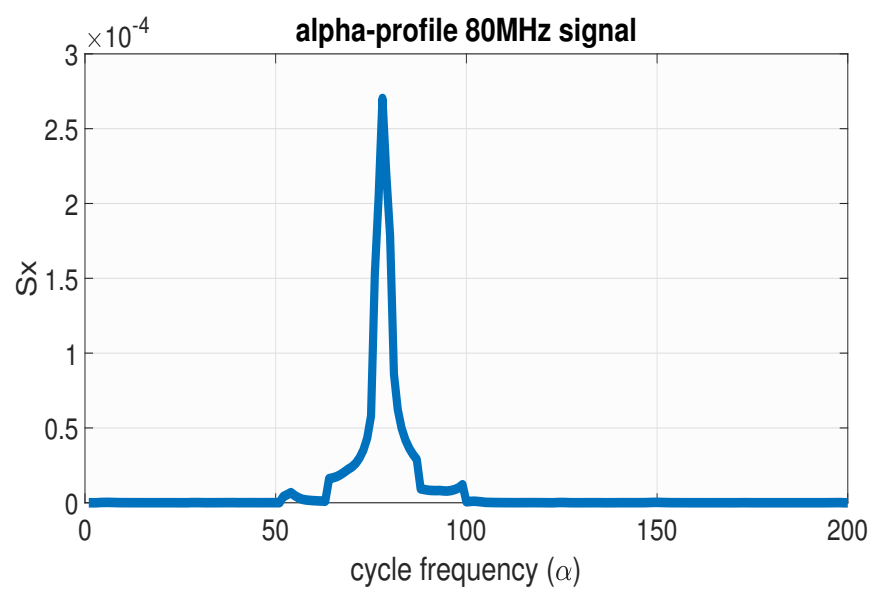

(c) $80 \mathrm{MHz}$ signal - HB

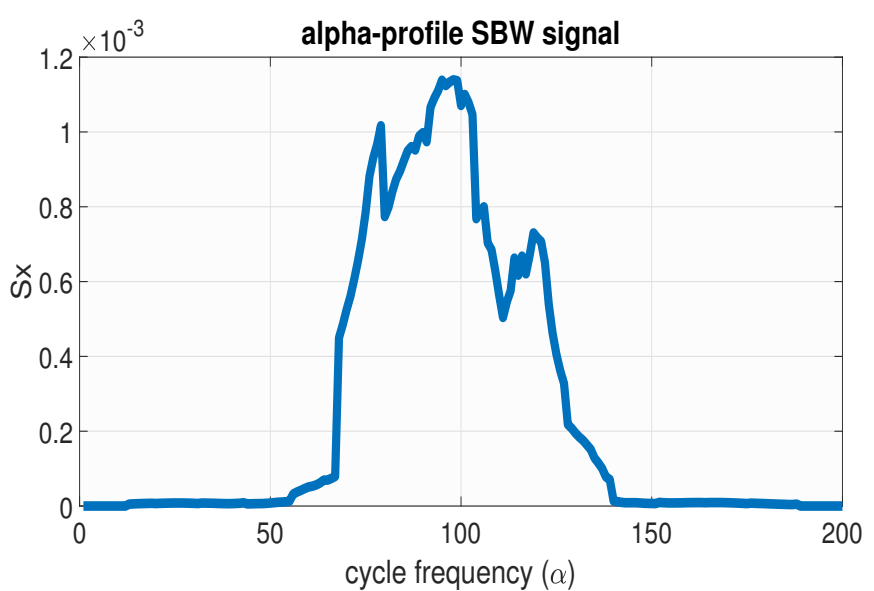

(b) SBW signal

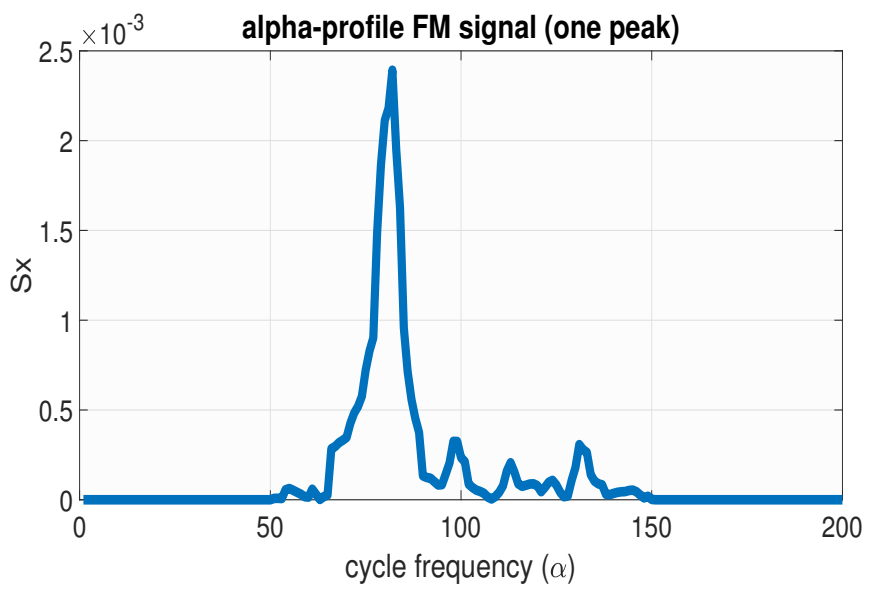

(d) one peak of the FM signal

Fig. 10: $\alpha$-profiles extracted from four different detected signals in the WB spectrum: a) baseband signal, b) SBW signal, c) interference at 80 $\mathrm{MHz}, \mathrm{d}$ ) one peak of the FM signal.

of the proposed algorithm in classifying the signals detected in the 0 $120 \mathrm{MHz}$ band-of-interest based on the $\alpha$-profile which is extracted from each detected waveform in the wideband spectrum and then fed to the neural network used as classifier.

Each detected waveform in the wideband spectrum, obtained after the pre-processing block in Sec. 3.2, is characterized by its own $\alpha$ profile which encompasses the cyclostationary feature. Specifically, each generated $\alpha$-profile consists of 200 cyclic frequency points which are the input of the ANN ( $I=200$ in Fig. 8). Figs. 10 (a)(d), show the $\alpha$-profiles for 4 of the different signals detected in the 0-120 MHz band: the 3-7 MHz signal, SBW signal, interference at $80 \mathrm{MHz}$, and one peak of the FM signal.

It is worth noticing, that the frequency content of a single cyclostationary signal is mainly characterized by both its fundamental frequency and its cyclic frequency (the former is usually higher than the latter for RF signals). It can be expected that the cyclic frequency is independent of the fundamental frequency and, consequently, the shape of the $\alpha$-profile of that signal does not change with the fundamental frequency and the ANN is able to classify the signal independently of the part of the spectrum in which it is detected. Future work will analyse signals located at RF, TVWS or ISM (industrial, scientific, and medical) band, for example, and the effective applicability of the proposed approach to signals in a spectrum beyond $120 \mathrm{MHz}$ could be demonstrated.

The ANN architecture employed to validate the analysis block of the CSI algorithm consists of with 10 neurons in the hidden layer $(N=10$ in Fig. 8). For our experiments, a dataset composed of 4.500 samples (500 for each configurations) is used in order to train (70\%), validate (15\%) and test (15\%) the ANN architecture. The overall performance can be observed in the 2-classes confusion matrix of Fig. 11 corresponding to the testing phase of the ANN which highlights a classification rate approximately equal to 1 . In this confusion matrix the tested waveforms are $15 \%$ of the all waveforms fed to the ANN in order to train it.

After having trained and tested the ANN with 4500 samples, further assessment of the performance is obtained with a different set of waveforms which are tested by using the trained neural network. Specifically, the performance of the system is evaluated for 1000 independent testing samples for each of the configuration with different carrier frequencies (51-61-71 MHz) and transmission powers $(7,4$, and $-3 \mathrm{dBm})$. The confusion matrices in Tab. 3 show the classification accuracy for the 9 different configurations. The proposed method based on the cyclostationary feature algorithm combined with an artificial neural network provides good performance with high classification rate in most of the configurations, even for low transmission power case.

A comparison with results in the literature is also presented. For example, in [29] spectrum sensing is performed through an energy detector to identify the occupied bands in the widedand spectrum. Center frequency, bandwidth and maximum value of magnitude are extracted for each of the identified narrowband waveforms. To perform classification, these parameters extracted from the identified waveforms are compared to parameters stored in a dataset containing pre-defined parameters of the FR and/or PM waveforms. This results in classification of each waveform as either FR or PM. 


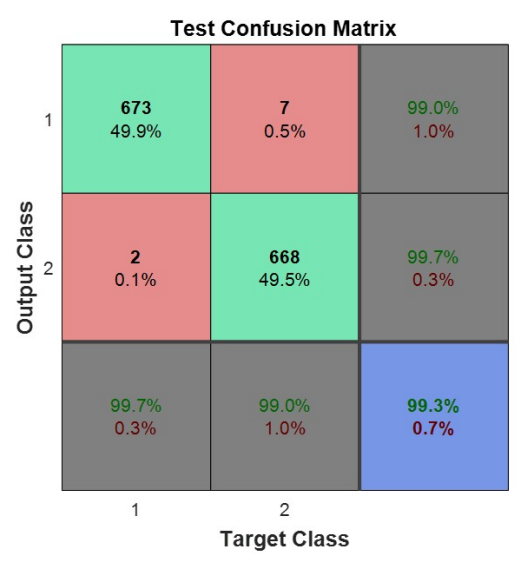

Fig. 11: Confusion matrix of the proposed ANN with two target and two output classes: $F R$ and $P M$.

For the sake of clarify, comparison is made on the percentage values in the confusion matrices.

In Tab. 4, the percentage values relative to all the configurations analysed in this manuscript, from $I$ to $I X$, are shown, while in Tab. 5 results from [29] can be seen where observed wideband spectrum, corresponding to both $61 \mathrm{MHz}$ and $71 \mathrm{MHz}$ centre frequencies, have been contemporary tested with 200 independent samples (or bursts). Two different feature vectors have been investigated, namely bandwidths of the detected waveforms in Tab. 5(a), while both bandwidths and magnitudes in Tab. 5(b). Specifically, different confusion matrices are obtained for each of the two type of feature vector by varying the number of bursts $\left(N_{b}\right)$ - over three levels $(1,3$, and 5$)$ which are averaged in order to increase the frequency resolution of the observed spectrum and to investigate its influence to the classification accuracy.

From comparison between these confusion matrices computed in [29] and the percentage values in Tab. 4 (considering only $61 \mathrm{MHz}$ and $71 \mathrm{MHz}$ ) obtained with the CFD and ANN implemented for the proposed algorithm, our results either outperform or are comparable with the ones in the literature, unless one specific case, for several reasons as discussed below. First of all, the percentage values are better or comparable in most of the cases. In addition, for the case of only bandwidths in the confusion matrices of Tab. 5, high PM and FR classification accuracies cannot be contemporary obtained with the same $N_{b}$ value, namely good accuracy for PM classification is reached with high $N_{b}$, while low $N_{b}$ values are recommended for better FR classification accuracy. While, in case of both bandwidths and magnitudes, results that could be comparable with our percentage values are reached with $N_{b}=5$. This produces an increased complexity of their algorithm and computational time as shown in [29]. Moreover, it should be noticed that by increasing $N_{b}$ the amount of testing bursts is dramatically reduced (from 200 to 40) which may reduce the reliability of the obtained classification rates. While in our case (which would correspond to $N_{b}=1$ ), firstly all the 1000 samples are used to perform the testing step with better reliability of classification results and, secondly, high FR classification and PM classification are contemporary obtained for each of the analysed configurations.

The only configuration in our results that produces minor PM classification accuracy than the other configurations, and then slightly smaller accuracy than the corresponding results for EDbased classifier, is $V I I I$ which corresponds to $-3 \mathrm{dBm}$ at $61 \mathrm{MHz}$. This could be due to a possible reduced quality of the SBW waveform in our newly created dataset for this configuration which could produce weaker performance of the classifier.

Nevertheless, even if the SNR level becomes low, the performance relative to configurations form $I V$ to $I X$ is still sufficient to guarantee good accuracy of the classification rate. It is also worth
Table 3 Confusion matrices with absolute values from testing on independent samples applied to the neural network; each configuration (from I to $I X$ ) has been tested separately.

\begin{tabular}{|c|c|c|c|c|c|c|}
\hline \multicolumn{7}{|c|}{ FULL POWER (7 dBm) } \\
\hline SIGNAL CLASS & FR & PM & FR & PM & \begin{tabular}{|l} 
I \\
FR
\end{tabular} & PM \\
\hline FR & 960 & 40 & 970 & 30 & 981 & 19 \\
\hline PM & 0 & 1000 & $\begin{array}{ll} & \end{array}$ & 999 & 0 & 1000 \\
\hline
\end{tabular}

HALF POWER (4 dBm)

\begin{tabular}{|c|c|c|c|c|c|c|}
\hline SIGNAL CLASS & FR & PM & FR & PM & FR & PM \\
\hline FR & 985 & 15 & 996 & 4 & 999 & 1 \\
\hline PM & 0 & 1000 & 0 & 1000 & 0 & 1000 \\
\hline
\end{tabular}

ONETENTH POWER (-3 dBm)

\begin{tabular}{|c|c|c|c|c|c|c|}
\hline SIGNAL CLASS & FR & PM & FR & PM & FR & PM \\
\hline FR & 997 & 3 & 997 & 3 & 997 & 3 \\
\hline PM & 20 & 980 & 88 & 912 & 0 & 1000 \\
\hline
\end{tabular}

Table 4 Confusion matrices with percentage values corresponding to the absolute values in Tab. 3 for all the analysed configurations, I-IX. Using the notation in the comparison with previous work, these confusion matrices are obtained with $N_{b}=1$.

\begin{tabular}{|c|c|c|c|c|c|c|}
\hline \multicolumn{7}{|c|}{ FULL POWER (7 dBm) } \\
\hline SIGNAL CLASS & FR & PM & FR & PM & FR & PM \\
\hline FR & $96.0 \%$ & $4.0 \%$ & $97.0 \%$ & $3.0 \%$ & $98.1 \%$ & $1.9 \%$ \\
\hline PM & $0 \%$ & $100 \%$ & $0.1 \%$ & $99.9 \%$ & $0 \%$ & $100 \%$ \\
\hline & \multicolumn{2}{|c|}{$\begin{array}{c}51 \mathrm{MHz} \\
\text { (I) }\end{array}$} & \multicolumn{2}{|c|}{$\begin{array}{c}61 M H z \\
\text { (II) }\end{array}$} & \multicolumn{2}{|c|}{$\begin{array}{c}71 \mathrm{MHz} \\
\text { (III) }\end{array}$} \\
\hline
\end{tabular}

\begin{tabular}{|c|c|c|c|c|c|c|}
\hline \multicolumn{7}{|c|}{ HALF POWER (4 dBm) } \\
\hline SIGNAL CLASS & FR & PM & FR & PM & FR & PM \\
\hline$\underline{\mathbf{F R}}$ & $98.5 \%$ & $1.5 \%$ & $99.6 \%$ & $0.4 \%$ & $99.9 \%$ & $0.1 \%$ \\
\hline PM & $0 \%$ & $100 \%$ & $0 \%$ & $100 \%$ & $0 \%$ & $100 \%$ \\
\hline
\end{tabular}

\begin{tabular}{|c|c|c|c|c|c|c|}
\hline \multicolumn{7}{|c|}{ ONETENTH POWER (-3 dBm) } \\
\hline SIGNAL CLASS & FR & PM & FR & PM & FR & PM \\
\hline FR & $99.7 \%$ & $0.3 \%$ & $99.7 \%$ & $0.3 \%$ & $99.7 \%$ & $0.3 \%$ \\
\hline PM & $2.0 \%$ & $98.0 \%$ & | $8.8 \%$ & $91.2 \%$ & $0 \%$ & $100 \%$ \\
\hline & \multicolumn{2}{|c|}{$\begin{array}{c}51 \mathrm{MHz} \\
(\mathrm{VII})\end{array}$} & \multicolumn{2}{|c|}{$\begin{array}{c}61 \mathrm{MHz} \\
\text { (VIII) }\end{array}$} & \multicolumn{2}{|c|}{$\begin{array}{l}71 \mathrm{MHz} \\
(I X)\end{array}$} \\
\hline
\end{tabular}

noticing that, unlike the previous work in [29], in this manuscript the proposed feature-based classifier is validated on a dynamic environment in which signals change either their carrier frequency or their transmission power (or both of them) in different cognitive cycles. Consequently, the training step is performed on a more complex dataset with a wider range of signal characteristics and shapes than 
Table 5 Confusion matrices with percentage values corresponding to the results in [29] obtained with ED-based classifier. Two different feature variables are considered: a) only bandwidth of each signal and b) bandwidth + magnitude for each signal. Bursts corresponding to two transmission frequencies, $61-71 \mathrm{MHz}$, are used together as input data. Each of these two tables consists of three different levels $(1,3$, and 5$)$ for the amount of bursts averaged $\left(N_{b}\right)$ to increase the frequency resolution.

61-71 MHz - only bandwidths [29]

\begin{tabular}{|c|c|c|c|c|c|c|c|}
\hline SIGNAL CLASS & FR & PM & ! & FR & PM & $\begin{array}{l}\text { FR } \\
\end{array}$ & PM \\
\hline$\underline{\mathbf{F}} \mathbf{R}$ & $95.5 \%$ & $4.5 \%$ & I & $59 \%$ & $41 \%$ & $15 \%$ & $85 \%$ \\
\hline PM & $35 \%$ & $65 \%$ & I & $7.6 \%$ & $92.4 \%$ & $0 \%$ & $100 \%$ \\
\hline
\end{tabular}

a)

61-71 MHz - both bandwidths and magnitudes [29]

\begin{tabular}{|c|cc:cc:cc|}
\hline SIGNAL CLASS & FR & PM & FR & PM & FR & PM \\
\hline FR & $100 \%$ & $0 \%$ & $100 \%$ & $0 \%$ & $100 \%$ & $0 \%$ \\
\hline PM & $38.5 \%$ & $61.5 \%$ & $18.2 \%$ & $81.8 \%$ & $7.5 \%$ & $92.5 \%$ \\
\hline
\end{tabular}

no. of bursts $\left(N_{b}\right)$ :

b)

in the previous work employing ED-based classifier in an almost stationary environment. This means that higher classification capability is required for dynamic scenarios.

From the literature, [30] proposes an interference mitigation algorithm for Spectrum Intelligence employing a naive Bayes classifier to discriminate FR from PM waveforms in a stationary environment. In Tab. 6, the corresponding results are shown in percentage values. Specifically, the SBW signal, which is considered as PM waveform, is transmitted with onetenth $(-3 \mathrm{dBm})$ power at $51 \mathrm{MHz}$ central frequency.

Different combinations of available features are used to evaluate the performance of the classifier. Namely, four combinations of bandwidth, maximum magnitude, and variance for each of the extracted waveform (both PM and FR) from the spectrum of interest as shown in the confusion matrices in Tab. 6: i) classification rates when the classifier combines all the three features, ii), iii), and iv) classification rates when classifier combines bandwidth and maximum magnitude, maximum magnitude and variance, and bandwidth and variance, respectively.

The naive Bayes classifier is trained with 50 bursts while additional 50 bursts, independent from the training data, are used for the testing step. For each burst there is just one PM signal (the SBW) and several FR waveforms. Consequently, the classifier is tested on 50 PM samples and almost 2000 FR samples.

By comparison with our results with onetenth power and $51 \mathrm{MHz}$, in the FR classification case the CFD and ANN-based algorithm produces better or comparable percentage classification rates than the naive Bayes-classifier in Tab. 6 for all the four combinations of features. Concerning the PM classification, the performance of our algorithm for configuration $V I I$, namely $-3 \mathrm{dBm} / 51 \mathrm{MHz}$, is not as good as in the other configurations and, consequently, lower values when compared with the percentage rates in Tab. 6. This has been discussed previously for configuration $V I I I$ and may be due to a possible reduced quality of the SBW waveform. In addition, it is worth noticing that for classifier in [30], only 50 samples corresponding to SBW vaweform in a stationary environment are used both during training step and during testing step which could produce unreliably classification rates. Again, dynamic scenarios require higher classification capability than stationary ones, which means that results in this paper could be underestimated with respect to the previous work.

Considering that, the newly implemented analysis block in our proposed work shows promising performance for the CSI processing in complex dynamic environments that also include signals with low SNR level.
Table 6 Confusion matrices in [30] for the parameter configuration $-3 \mathrm{dBm}$ / $51 \mathrm{MHz}$ (corresponding to configuration $V I I$, if notation in this paper is considered). Combinations of the considered features are: i) bandwidth + amplitude + variance ii) bandwidth + amplitude iii) amplitude + variance iv) bandwidth + variance.

$-3 \mathrm{dBm} / 51 \mathrm{MHz}[30]$

\begin{tabular}{|c|c|c|c|c|}
\hline SIGNAL CLASS & FR & PM & FR & PM \\
\hline FR & $99.1 \%$ & $0.9 \%$ & $99.8 \%$ & $0.2 \%$ \\
\hline PM & $0 \%$ & $100 \%$ & $0 \%$ & $100 \%$ \\
\hline
\end{tabular}

\begin{tabular}{|c|c|c|c|}
\hline \multicolumn{4}{|c|}{$-3 \mathrm{dBm} / 51 \mathrm{MHz}$ [30] } \\
\hline FR & PM & FR & PM \\
\hline $98.5 \%$ & $1.5 \%$ & $99.1 \%$ & $0.9 \%$ \\
\hline $4.0 \%$ & $96 \%$ & $0 \%$ & $100 \%$ \\
\hline
\end{tabular}

\section{Conclusion and future work}

In the manuscript, a new AI-enabled cognitive radio framework is proposed as part of a Cyclic Spectrum Intelligence algorithm to enable interference mitigation in wideband radios. The CSI algorithm is based on a Cognitive Cycle consisting of 5 blocks: Sensing, Processing, Analysis, Learning, and Acting. The Cognitive Cycle interacts with the radio environment through the sensing block which obtains instantaneous spectrum data in the band of interest and the acting block whose task is to decide and change the transmission parameters such as the carrier frequency and the transmission power. The main novelty introduced by our work is the analysis block. After having identified occupied channels and spectrum holes in the observed wideband signal, the stationary spectral analysis is performed, based on the cyclostationary feature of modulated signals, to extract the $\alpha$-profile from the SCF of the detected signals. Afterwards, the extracted $\alpha$-profile of a number of samples of the different waveforms detected in the wideband spectrum are fed to a pre-trained ANN to classify the waveforms as "friendly" or "potential malicious". In addition, a software defined radio testbed has been employed to generate an experimental dateset and validate the analysis block. Real data consists of spectrum measurements in the 0-120 $\mathrm{MHz}$ in which there are different signals including the SBW signal whose carrier frequency and transmission power are given different values. Consequently, a large amount of experimental data in a dynamic environment is collected and stored in a complex dataset with a wide range of signal characteristics and shapes, and organized for off-line research applications. Nine different configurations of these values have been used in the validation step of the proposed analysis block.

Results show high classification rate in most of the configurations even for low transmission power case making the new block based on cyclostationary feature detector with artificial neural network a promising methodology for the CSI processing in complex dynamic environments that also include signals with low SNR level. In support of this claim, a comparison with existing works has also been carried out. The main drawback related to these two previous approaches is that the variability of both the hand-crafted features and noise do not guarantee accurate classification especially at low SNR level. While, the proposed approach overcomes that thanks to better extracted features and the neural network classifier. In one of the considered previous works, an ED-based classifier was employed to differentiate FR class from PM class in an almost stationary environment. Our algorithm for dynamic environments proposed in this paper produces better (in some cases) and comparable (in other cases) results than the ED-based algorithm. However, it is worth noticing that these results are obtained without resorting to multiple-bursts average (namely with $N_{b}=1$ ) necessary to improve the performance of the classifier in the previous work. This avoids, 
consequently, an increased complexity and computational time as well as a reduced data-samples set for both training and testing which could reduce the reliability of the resulting classification rates.

A naive Bayes-based classifier has also been previously investigated in a stationary spectrum. Although our CFD and ANN-based algorithm is trained and tested on a large number of samples from a dynamic scenario, it still produces better or comparable classification rates with respect to the existing approach for the FR class. Concerning the PM class, a possible loss of quality in the SBW signal in the newly created dataset may be the cause of obtaining not as good results as the ones obtained for all the other configurations. However, since the Bayes-based classifier is trained and tested by using much less samples concerning the PM class, the corresponding results may be not enough accurate. In addition, our classifier is analysed in a dynamic environment which means higher classification capability is required.

Future work includes analysis of fully autonomous systems capable of dynamically access the spectrum in a cognitive radio framework for applications such as PHY-layer security against jamming attacks as well as TVWS. The effective applicability of the proposed approach to signals in a spectrum beyond $120 \mathrm{MHz}$ can also be investigated and demonstrated, although it is expected that the shape of the $\alpha$-profile of a signal is independent of its fundamental frequency. In addition, Stealthy Jammer Detection Algorithm investigated in [46] and in [47] can be validated on the experimental dataset described in this manuscript.

\section{References}

1 Friston, K., Sengupta, B., Auletta, G.: 'Cognitive dynamics: From attractors to active inference', Proceedings of the IEEE, 2014, 102, (4), pp. 427-445

2 Haykin, S.: 'Cognitive dynamic systems: Radar, control, and radio [point of view]', Proceedings of the IEEE, 2012, 100, (7), pp. 2095-2103

3 Office of Communications (Ofcom), Feb. 2015, 'Implementing tv white spaces, [online], available: http://stakeholders.ofcom.org.uk/binaries/consultations/whitespace-coexistence/statement/tvws-statement.pdf'

4 Holland, O., Ping, S., Aijaz, A., Chareau, J., Chawdhry, P., Gao, Y., et al.: 'To white space or not to white space: That is the trial within the ofcom tv white spaces pilot', In: 2015 IEEE International Symposium on Dynamic Spectrum Access Networks (DySPAN), 2015, pp. 11-22

5 Qin, Z., Gao, Y., Parini, C.G.: 'Data-assisted low complexity compressive spectrum sensing on real-time signals under sub-nyquist rate', IEEE Transactions on Wireless Communications, 2016, 15, (2), pp. 1174-1185

6 Qin, Z., Gao, Y., Plumbley, M.D., Parini, C.G.: 'Wideband spectrum sensing on real-time signals at sub-nyquist sampling rates in single and cooperative multiple nodes', IEEE Transactions on Signal Processing, 2016, 64, (12), pp. 3106-3117

7 Ma, Y., Gao, Y., Liang, Y., Cui, S.: 'Reliable and efficient sub-nyquist wideband spectrum sensing in cooperative cognitive radio networks', IEEE Journal on Selected Areas in Communications, 2016, 34, (10), pp. 2750-2762

8 Martin, J.H., Dooley, L.S., Wong, K.C.P.: 'New dynamic spectrum access algorithm for tv white space cognitive radio networks', IET Communications, 2016, 10, (18), pp. 2591-2597

9 Lin, S., Kong, L., Gao, Q., Khan, M.K., Zhong, Z., Jin, X., et al.: 'Advanced dynamic channel access strategy in spectrum sharing 5g systems', IEEE Wireless Communications, 2017, 24, (5), pp. 74-80

10 Gao, Y., Qin, Z., Feng, Z., Zhang, Q., Holland, O., Dohler, M.: 'Scalable and reliable iot enabled by dynamic spectrum management for $\mathrm{m} 2 \mathrm{~m}$ in lte-a', IEEE Internet of Things Journal, 2016, 3, (6), pp. 1135-1145

11 Fiaschetti, A., Noll, J., Azzoni, P., Uribeetxeberria, R.: 'Measurable and Composable Security, Privacy, and Dependability for Cyberphysical Systems: The SHIELD Methodology', CRC Press, 2017

12 Qin, Z., Gao, Y., Plumbley, M.D.: 'Malicious user detection based on low-rank matrix completion in wideband spectrum sensing', IEEE Transactions on Signal Processing, 2018, 66, (1), pp. 5-17

13 Mohammadi, J., Stańczak, S., Zheng, M.: 'Joint spectrum sensing and jamming detection with correlated channels in cognitive radio networks', In: 2015 IEEE International Conference on Communication Workshop (ICCW), 2015, pp. 889894

14 Chang, G.Y., Wang, S.Y., Liu, Y.X.: 'A jamming-resistant channel hopping scheme for cognitive radio networks', IEEE Transactions on Wireless Communications, 2017, 16, (10), pp. 6712-6725

15 Dabčević, K., Marcenaro, L. Regazzoni, C.S. 'Security in cognitive radio networks', In: Evolution of Cognitive Networks and Self-Adaptive Communication Systems, 2013, pp. 301-335, IGI Global

16 Haykin, S., Thomson, D., , Reed, J.: 'Spectrum sensing for cognitive radio', Proceedings of the IEEE, 2009, 97, (5), pp. 849-877

17 Chin, W.L., Li, J.M., Chen, H.H. 'Low-complexity energy detection for spectrum sensing with random arrivals of primary users', IEEE Transactions on Vehicular Technology, 2015, 65, (2), pp. 947-952

18 Kozowski, S.: 'Implementation and verification of cyclostationary feature detector for dvb-t signals', IET Signal Processing, 2016, 10, (2), pp. 162-167
19 Xinzhi, Z., Feifei, G., Rong, C., Tao, J.: 'Matched filter based spectrum sensing when primary user has multiple power levels', China Communications, 2015, 12, (2), pp. 21-31

20 Zhao, Y., Wu, Y., Wang, J., Zhong, X., Mei, L.: 'Wavelet transform for spectrum sensing in cognitive radio networks', In: International Conference on Audio, Language and Image Processing (ICALIP), Shanghai, China, 2014, pp. 565-569

21 Gardner, W., Cadzow, J.A.: 'Statistical spectral analysis: a nonprobabilistic theory', Applied Optics, 1990, 29, pp. 1399

22 Roberts, R.S., Brown, W.A., Loomis, H.H.: 'Computationally efficient algorithms for cyclic spectral analysis', IEEE Signal Processing Magazine, 1991, 8, (2), pp. 38-49

23 Nawaz, T., Mughal, M.O., Marcenaro, L., Regazzoni, C.S.: 'Exploiting cyclic features for jammer detection in wide-band cognitive radios', In: WInnCommEurope' 15, Erlangen, Germany, 2015

24 Wu, Z., Like, E., Chakravarthy, V.: 'Reliable modulation classification at low snr using spectral correlation', In: 4th IEEE Consumer Communications and Networking Conference, 2007, pp. 1134-1138

25 Like, E., Chakravarthy, V., Husnay, R., Wu, Z.: 'Modulation recognition in multipath fading channels using cyclic spectral analysis', In: IEEE Global Telecommunications Conference (GLOBECOM), 2008, pp. 1-6

26 Mughal, M.O., Dabčević, K., Marcenaro, L., Regazzoni, C.S.: 'Compressed sensing based jammer detection algorithm for wide-band cognitive radio networks', In: Compressed Sensing Theory and its Applications to Radar, Sonar and Remote Sensing (CoSeRa), 2015 3rd International Workshop on, 2015, pp. 119-123

27 Mughal, M.O., Nawaz, T., Marcenaro, L., Regazzoni, C.S.: 'Cyclostationary-based jammer detection algorithm for wide-band radios using compressed sensing', In: 2015 IEEE Global Conference on Signal and Information Processing (GlobalSIP), 2015, pp. 280-284

28 Yucek, T., Arslan, H.: 'A survey of spectrum sensing algorithms for cognitive radio applications', IEEE Communications Surveys Tutorials, 2009, 11, (1), pp. 116-130

29 Dabčević, K., Mughal, M.O., Marcenaro, L., Regazzoni, C.S. 'Spectrum intelligence for interference mitigation for cognitive radio terminals', In: Wireles Innovation Forum European Conference on Communications Technologies and Software Defined Radio (WInnComm- Europe), Rome, Italy, 2014

30 Dabčević, K., Mughal, M.O., Marcenaro, L., Regazzoni, C.S.: 'Cognitive radio as the facilitator for advanced communications electronic warfare solutions', Journal of Signal Processing Systems, 2016, 83, (1), pp. 29-44

31 Nawaz, T., Campo, D., Mughal, M.O., Marcenaro, L., Regazzoni, C.S.: 'Jammer detection algorithm for wide-band radios using spectral correlation and neural networks', In: 2017 13th International Wireless Communications and Mobile Computing Conference (IWCMC), Valencia, Spain, 2017

32 Nafkha, A., Naoues, M., Cichon, K., Kliks, A.: 'Experimental spectrum sensing measurements using usrp software radio platform and gnu-radio', In: 2014 9th International Conference on Cognitive Radio Oriented Wireless Networks and Communications (CROWNCOM), 2014, pp. 429-434

33 Nafkha, A., Aziz, B., Naoues, M., Kliks, A.: 'Cyclostationarity-based versus eigenvalues-based algorithms for spectrum sensing in cognitive radio systems: Experimental evaluation using gnu radio and usrp', In: 2015 IEEE 11th International Conference on Wireless and Mobile Computing, Networking and Communications (WiMob), 2015, pp. 310-315

34 Dabčević, K., Marcenaro, L., Regazzoni, C.S.: 'Spd-driven smart transmission layer based on a software defined radio test bed architecture', In: 4th International Conference on Pervasive and Embedded Computing and Communication Systems (PECCS) - Lisbon, Portugal, 2014

35 Bhagate, S.V., Patil, S.: 'Maximizing spectrum utilization in cognitive radio network', In: 2017 International Conference on Big Data, IoT and Data Science (BID), 2017, pp. 82-90

36 Petty, V.R., Rajbanshi, R., Datla, D., Weidling, F., DePardo, D., Kolodzy, P.J., et al.: 'Feasibility of dynamic spectrum access in underutilized television bands', In: 2007 2nd IEEE International Symposium on New Frontiers in Dynamic Spectrum Access Networks, 2007, pp. 331-339

37 April, E.: 'Ad-a258 307 the advantage of cyclic spectral analysis (u)', technical note, Defence Research Establishment Ottawa, October, 1991

38 Gardner, W., Franks, L.: 'Characterization of cyclostationary random signal processes', IEEE Transactions on Information Theory, 1975, 21, (1), pp. 4-14

39 Møller, M.F.: 'A scaled conjugate gradient algorithm for fast supervised learning', Neural networks, 1993, 6, (4), pp. 525-533

40 Gardner, W.: 'Spectral correlation of modulated signals: Part i - analog modulation', IEEE Transactions on Communications, 1987, 35, (6), pp. 584-594

41 Gardner, W., Brown, W., Chen, C.K.: 'Spectral correlation of modulated signals Part ii - digital modulation', IEEE Transactions on Communications, 1987, 35, (6), pp. 595-601

42 Gardner, W.A.: 'Statistical spectral analysis: A nonprobabilistic theory', Englewood Cliffs, N.J: Prentice Hall, 1988

43 Gardner, W.A.: 'Exploitation of spectral redundancy in cyclostationary signals', IEEE Signal Processing Magazine, 1991, 8, (2), pp. 14-36

44 Yousry1, H., Newagy, F., ElRamly, S., Ibrahim, M.: 'Wireless microphone sensing using cyclostationary detector', In: 2012 Innovations on Communication Theory (INCT2012), 2012

45 Alfaqawi, M.I.M., Chebil, J., Habaebi, M.H., Datla, D.: 'Wireless distributed computing for cyclostationary feature detection', Digital Communications and Networks, 2016, 2, (1), pp. $47-56$

46 Nawaz, T., Marcenaro, L., Regazzoni, C.S.: 'Defense against stealthy jamming attacks in wide-band radios: A physical layer approach', In: IEEE Global Conference on Signal and Information Processing (GlobalSIP' 17), Montreal, Canada, 2017

47 Nawaz, T., Marcenaro, L., Regazzoni, C.S.: 'Stealthy jammer detection algorithm for wide-band radios: A physical layer approach', In: IEEE 10th International Workshop on Selected Topics in Wireless and Mobile computing (STWiMob' 17), Rome, Italy, 2017 